\section{Revista Brasileira de Administração Científica}

Brazilian Journal of Scientific Administration

Abr a Jun $2021-$ v.12 - n.2

\title{
O patriarcado como barreira no desenvolvimento do empreendedorismo feminino
}

Este artigo teve como objetivo apresentar como o patriarcado tem sido discutido na literatura de administração. Para tanto, optou-se pela realização de uma revisão sistemática da literatura com técnicas de análise de conteúdo e classificação hierárquica descendente. No total foram estudados 79 artigos provenientes da base Web of Science, no período de 1987 a 2020. Os resultados demonstram que os artigos foram agrupados de acordo com as seguintes categorias: a) as questões do feminismo e a injustiça dos direitos das mulheres; b) as questões associadas à contextos em que evidenciam a desigualdade social e econômica, cultural e religiosa; c) as questões que abordam o conceito do feminismo e que as questões de desigualdade de gênero e d) as questões do equilíbrio da vida familiar, doméstica, religiosa e a vida profissional da mulher. Percebe-se o patriarcado como barreira no desenvolvimento da vida profissional das mulheres.

Palavras-chave: Patriarcado; Gênero; Empreendedorismo; Patriarcado Público; Patriarcado Particular.

\section{Patriarchy as a barrier in the development of female entrepreneurship}

This article aimed to present how patriarchy has been discussed in the management literature. For that, we opted for a systematic review of the literature with
techniques of content analysis and descending hierarchical classification. In total, 79 articles from the Web of Science database were studied, from 1987 to 2020 .
The results show that the articles were grouped according to the following categories: a) the issues of feminism and the injustice of women's rights; b) issues
associated with the contexts in which they demonstrate social and economic, cultural and religious inequality; c) issues that address the concept of feminism and
that issues of gender inequality; and d) issues of balance in family, domestic, religious life and the professional life of women. Patriarchy is perceived as a barrier
in the development of women's professional lives.

Keywords: Patriarchate; Genre; Entrepreneurship; Public Patriarchate; Private Patriarchate.

Topic: Empreendedorismo

Reviewed anonymously in the process of blind peer.
Received: 07/04/2021

Approved: 04/06/2021
Simone Grecco Sanches Sueiro (iD)

Universidade Nove de Julho, Brasil

http://lattes.cnpq.br/3472078467877217

http://orcid.org/0000-0003-1511-1726

simonegrecco.sanches@gmail.com
Referencing this:

SUEIRO, S. G. S.. O patriarcado como barreira no desenvolvimento do empreendedorismo feminino. Revista Brasileira de Administração Científica, v.12, n.2, p.83-89, 2021. DOI:

http://doi.org/10.6008/CBPC2179-684X.2021.002.0007 


\section{INTRODUÇÃO}

A discriminação de gênero no local de trabalho tem sido um fenômeno universal, com alguns locais de trabalho e países piores que outros (Hodgkinson et al., 2009; Burke \& Richardsen, 2016). No entanto, as mulheres também continuam lutando contra o patriarcado e as normas patriarcais em casa e no local de trabalho (ADISA et al., 2019; ADISA et al., 2019; MAKAMA, 2013).

Dentro do escopo deste estudo as pesquisas sobre patriarcado destaca o papel da mulher na sociedade e nas organizações, as barreiras sociais e estruturais (AHMAD et al., 2011; BIKA, 2012; BOUTILIER et al., 2009; NAGUIB et al., 2015; SAIFUDDIN et al., 2019) Essas barreiras são normalmente abordadas no contexto de países que seguem normas socioculturais de uma cultura sob a dominação do homem, principalmente em países da Ásia (AFIOUNI et al., 2014; KAMLA, 2014; MASOOD et al., 2020; PRIMECZ et al., 2019; SAIFUDDIN et al., 2019; SALEM et al., 2019; YAMAK et al., 2016) e da África (ADISA et al., 2019; ADISA et al., 2019; HENNEKAM, 2015; MUSHFIQUR et al., 2018) identificando que poucos são os estudos em países do Oriente (BOUTILIER et al., 2009; NETTO et al., 2020).

Assim, este estudo levanta um aspecto que problematiza a questão do patriarcado, evidenciando seu nível de interferência na vida profissional da mulher, em diferentes contextos, como em países que valorizam a cultura de dominação patriarcal privada, a crença religiosa, crise econômica e crise política. Este artigo tem como objetivo apresentar como o patriarcado tem sido discutido na literatura de administração no período de 1987 a 2020. Os objetivos específicos para a realização deste estudo consistiram em mapear as teorias, métodos, tipologias e demais aspectos que pudesses sintetizar e esclarecer o que há no campo científico sobre o patriarcado.

\section{REVISÃO TEÓRICA}

O patriarcado é um fenômeno social estrutural que afeta as mulheres em casa (patriarcado particular) e em seus vários locais de trabalho (patriarcado público). Esses autores descrevem um sistema de 'patriarcado clássico', no qual as mulheres aceitam seu status subordinado em troca de proteção física, segurança econômica e respeitabilidade social. Já um outro autor diz que patriarcado é um conceito feminista usado para entender e analisar a organização sistemática da supremacia masculina e subordinação feminina, ou o domínio da mulher pelo homem

O patriarcado é um fenômeno social estrutural que afeta as mulheres tanto na vida pessoal quanto na vida profissional. As pessoas tendem a se comportar em organizações de trabalho baseadas em roteiros aprendidos por meio da socialização organizacional, experiência de trabalho e uma multiplicidade de outros fatores inter-relacionados, como crenças e normas culturais, dificultando a coexistência pacífica como uma comunidade de indivíduos iguais (ADISA et al., 2019)

É um sistema que encoraja a liderança, a dominação e o poder masculino no qual as mulheres estão sujeitas à dependência econômica, violência, domesticação e periféricos da tomada de decisões. Impõe estruturas que categorizam alguns tipos de trabalho como "trabalho de homens" e outros como "trabalho 
de mulheres". Desigualdade de gênero, sexismo e dominação masculina, entre outras coisas, são características de uma sociedade patriarcal (ADISA et al., 2019).

O patriarcado implica que a autoridade é investida no homem como chefe da família. Isso significa que ele supervisiona a propriedade e os ganhos da família e controla as preferências da família por trabalho, lazer e assuntos gerais da família. No entanto, diferentes culturas atribuem diferentes graus de significância a essas questões (ADISA et al., 2019)

\section{METODOLOGIA}

Inicialmente, foi realizada uma busca sem restrições relacionadas às áreas acadêmicas, revistas, tipo de trabalho ou datas de publicação com o intuito de gerar a base de dados inicial de artigos publicados sobre patriarcado. A base de dados escolhida para a coleta de dados foi o Web of Science, visto que esta plataforma é composta por dez índices que abarcam informações coletadas de diversos periódicos, livros, conferências e outros materiais de natureza acadêmica. O parâmetro inicial de busca foram as palavras 'patriarchy', abrangendo a pesquisa para todos os tipos de documentos ao longo dos anos.

No processo de busca resultaram 76 artigos que foram considerados para realização da análise na íntegra. Entre esta amostra, 11 artigos foram publicados em um único periódico (Gender work and organization), que parece ser uma das fontes mais relevantes no tema estudado.

Com o apoio do software Iramuteq, possibilitou desenvolver a análise de classificação hierárquica descendente. A Classificação Hierárquica Descendente (CHD) possibilitou a classificação dos segmentos de texto em função dos seus respectivos vocabulários e o conjunto deles é repartido com base na frequência das formas reduzidas.

\section{RESULTADOS E DISCUSSÃO}

A figura 1 é uma representação gráfica simples, da qual agrupa as palavras de acordo com a frequência. Desta forma, percebe-se que o tema, patriarcado está sendo discutido na área da administração relacionando estudos que contenham a palavra mulher, gênero, feminino e trabalho.

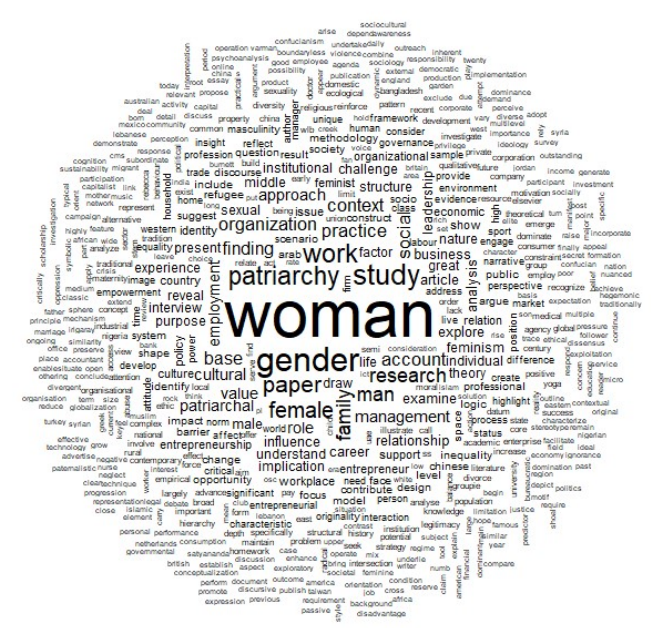

Figura 1: Nuvem de palavras a partir dos resumos dos artigos. 
A partir dos dados obtidos desta pesquisa foi possível visualizar o cenário das produções científicas internacionais na área de administração a respeito do tema Patriarcado. Neste primeiro momento, os 76 artigos foram classificados no que tange à semelhanças e diferenças, de acordo com o exposto na figura 2 .

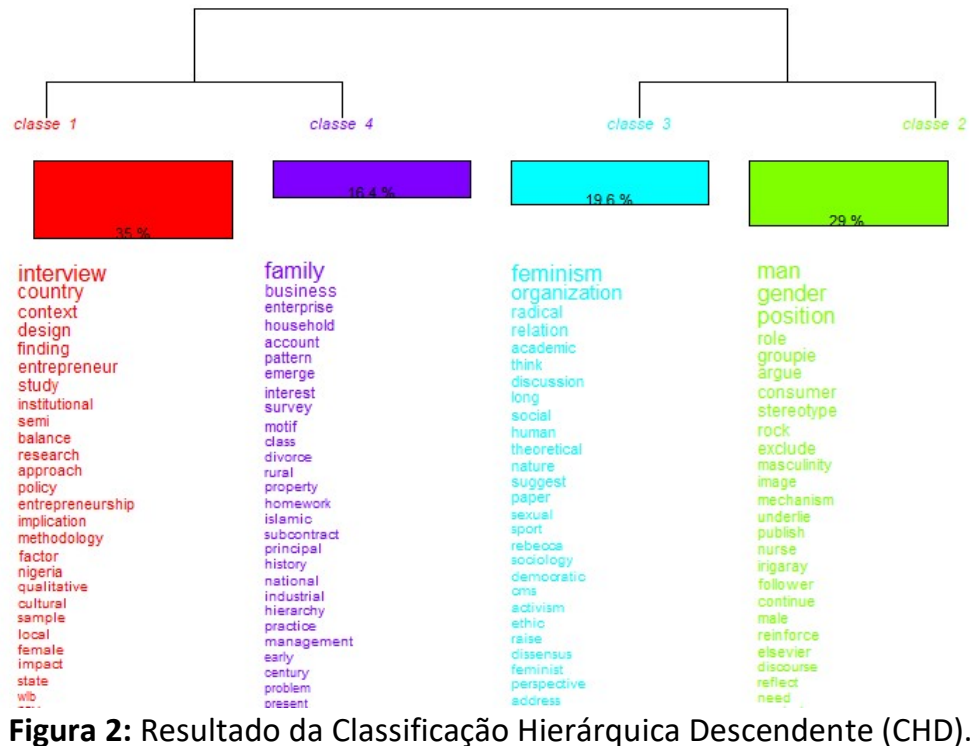

Como é possível notar, o tema tem sido abordado na literatura qualitativamente por meio de entrevistas, de forma que permita discussão quanto a maneira teórica quanto teórico-empírico.

De acordo com a porcentagem de vezes que o assunto se apresenta, tem-se, em primeiro plano, a classe 1 , que apresentou frequência de $35 \%$ do aparecimento deste grupo de palavras, que fica evidenciado que patriarcado está sendo pesquisado por meio de entrevistas. A influência do patriarcado associado a contextos em que evidenciam a desigualdade social e econômica, deixando claro que a cultura do país seja o principal influenciador do patriarcado na vida profissional das mulheres.

Nessa classe foi encontrado artigos em que os autores encontraram o empreendedorismo como sendo a única forma que as mulheres encontram para trabalhar, pois dentro das organizações as mulheres sofrem pela desigualdade de gênero.

O segundo grupo de palavras, com $16,4 \%$ em termos de frequência, foi a classe 4 , que contemplou a questão do equilíbrio da vida familiar, doméstica, religiosa e a vida profissional da mulher. Em generalizações monoculturais abrangentes sobre como influenciam a prática gerencial (FORSTER et al., 2014)

Em terceiro lugar tem-se a classe 3, que representou $19,6 \%$ do grupo de palavras em questão. É possível notar que nesse aspecto os artigos abordam o patriarcado por meio do conceito do feminismo, que no decorrer nos anos de tornou uma linha de pesquisa e que autores discutem a desigualdade de gênero preconizando o feminino, como o artigo da Bryant (1993) que abordam questões do patriarcado, raça, etnia, desvio de ética, economia para obter uma compreensão social além da comunidade acadêmica a sociedade passou a discutir o tema se envolvendo com causas e eventos feministas.

Na sequência, com $29 \%$ de frequência, apresenta-se a classe 2 . Através dela é possível compreender que o patriarcado e sua forma de caracterização e tipologia são discutidos em forma de gênero, em comparação com o homem evidenciando que existe gaps para estudos dentro desse tema. 
O Iramuteq gera também uma imagem que evidencia como as classes se relacionam. Desta forma, percebe- se que as classes 2 e 3 não estão tão divididos como nas demais, significando que na literatura, não há uma divisão clara quanto aos estudos que focam no gênero masculino e nos estudos que procurando entender o gênero feminino dentro do patriarcado.

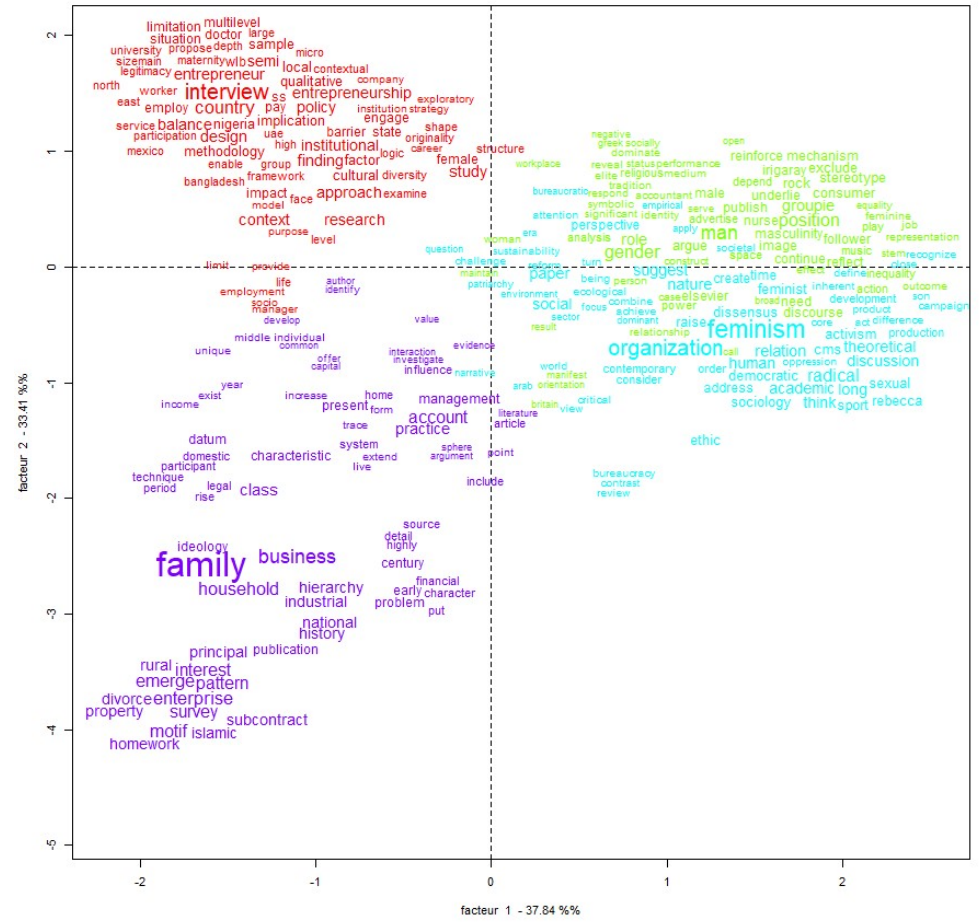

Figura 3: Resultado da Análise Fatorial Exploratória (AFE) com as classes da CHD.

Indo além, por meio de uma análise de similitude, também realizada pelo software Iramuteq, fica possível notar, pela figura 3, quais são os assuntos presentes nos artigos que se associam tematicamente, de modo como as classes se comportam.

Dessa forma, em complemento às demais análises já apresentadas neste estudo, fica evidenciado que os estudos sobre patriarcado associam as desigualdades sociais, econômicas, culturais sobre a vida profissional da mulher. Já a classe 2 traz artigos que tratam sobre o feminismo e os movimentos feministas evidenciado as discussões do patriarcado. Assim sendo, os estudos sobre patriarcado, principalmente os mais recentes, 2019 e os pertencem à classe 1 buscam evidências na cultura como alicerce às barreiras que as mulheres enfrentam.

\section{CONCLUSÕES}

A área de estudos sobre patriarcado na administração está em um movimento de retomada por um movimento na busca concreta da problematização do tema e pelas alternativas e as barreiras enfrentadas pelas mulheres, em suas vivencias bem como o impacto que propicia no contexto social e organizacional.

O campo do conhecimento está em evolução no contexto das organizações, pois a temática tem se apoiado em outras áreas, já consolidadas e que contribui na procura com a construção teórica, possibilitando uma melhor compreensão e expansão do conhecimento. Desta forma, a área de estudos sobre o feminismo está se consolidando no conhecimento das áreas das ciências sociais. 
Investigar a evolução das pesquisas sobre o patriarcado tem se mostrado relevante diante do crescente número de estudos sobre o tema, em especial com relação aos impactos que os gêneros parecem ter neste fenômeno. Foi realizada uma revisão sistemática da literatura para entender melhor esta evolução, pois este estudo permitiu conhecer o estado da arte deste determinado tema.

Com base nesses estudos, esse artigo teve por objetivo levantar e analisar a produção científica do período de 1987 a 2020 sobre patriarcado na administração. Observou-se que a quantidade de artigos encaminhados para as áreas de conhecimento, nos últimos anos, vem crescendo e abrindo perspectivas de ampliação do conhecimento gerando assim, alternativas para o desenvolvimento de novas pesquisas, haja visto que o número de artigos publicados pulo de 4 em 2018 para 19 em 2019.

É relevante mencionar que os artigos publicados em 2019 são discutidos como a cultura, a política, a religião e a desigualdade social econômica impactam na vida profssional da mulher após esses 30 anos de estudos sobre patriarcado. Entretanto, parece ser esse o tema deverá ser mais explorado na busca de consolidar os achados em pesquisas nos campos de estudo em administração.

Desta forma, o caminho da produção teórica é fundamental para expandir e ir se fazendo a partir de suas práticas e pesquisas, das demais áreas do conhecimento, como os estudos do feminismo e não somente em nível internacional como este estudo, mas também em nível nacional.

\section{REFERÊNCIAS}

ADISA, T. A.; ABDULRAHEEM, I.; ISIAKA, S. B.. Patriarchal hegemony: investigating the impact of patriarchy on women's work-life balance. Gender in Management, v. 34 n.1, p.19-33, 2019.

ADISA, T. A.; COOKE, F. L.; IWOWO, V.. Mind your attitude: the impact of patriarchy on women's workplace behaviour. Career Development International, v.25, n.2, p.146-164, 2019.

AFIOUNI, F.; RUËL, H.; SCHULER, R.. HRM in the Middle East: Toward a greater understanding. International Journal of Human Resource Management, v.25, n.2, p.133-143, 2014

AHMAD, H. M.; NAIMAT, S.. Networking and women entrepreneurs. Beyond Patriarchal Traditions, v.5, n.14, p.5784-5791, 2011.

BIKA, Z.. Entrepreneurship \& regional development: an international journal entrepreneurial sons, patriarchy and the colonels' experiment in thessaly, rural Greece.

Entrepreneurship and Regional Development, v.24, p.235257, 2012.

BOUTILIER, R.; BOUTILIER, R.. Globalization and the careers of mexican knowledge workers: an exploratory study of employer and worker adaptations. Journal of Business Ethics, v.88, p.319-333, 2009.

BRYANT, J. E.. Sport Management and the Interdependence with sport sociology: sport as a social product. Journal of Sport Management, v.7, n.3, p.194-198, 1993. DOI:

https://doi.org/10.1123/ism.7.3.194
FORSTER, G.; FENWICK, J.. The influence of Islamic values on management practice in Morocco. European Management Journal, v.33, n.2, p.1-14, 2014. DOI: https://doi.org/10.1016/j.emj.2014.04.002

HENNEKAM, S.. Career success of older workers: the influence of social skills and continuous learning ability. Journal of Management Development, v.34, n.9, p.11131133, 2015.

HODGKINSON, G. P.; SADLER-SMITH, E.; BURKE, L. A.; CLAXTON, G.; SPARROW, P. R.. Intuition in organizations: implications for strategic management. Long Range Planning, v.42, n.3, p.277-297, 2009.

KAMLA, R.. Modernity, space-based patriarchy and global capitalism: implications for Syrian women accountants Modernity, space-based patriarchy and global capitalism: implications for Syrian women accountants. Accounting and Business Reserach, v.44, n.6, p.603-629, 2014.

MAKAMA, G. A.. Patriarchy and gender inequality in nigeria: the way forward. European Scientific Journal, v.9, n.17, p.115-144, 2013.

MASOOD, A.; NISAR, M. A.. Crushed between two stones: Competing institutional logics in the implementation of maternity leave policies in Pakistan. Gender, Work and Organization, v.27, n.6, p.1103-1126, 2020. DOI: https://doi.org/10.1111/gwao.12448

MUSHFIQUR, R.; MORDI, C.; ORUH, E. S.; NWAGBARA, U.; MORDI, T.; TURNER, I.. The impacts of work-life-balance (WLB) challenges on social sustainability The experience of 
Nigerian female medical doctors. Employee Relations, v.40, n.1, 2018. DOI: http://doi.org/10.1108/ER-06-2017-0131

NAGUIB, R.; JAMALI, D.. Female entrepreneurship in the UAE: a multi-level integrative lens. Gender in Management, v.30, p.135-161, 2015.

NETTO, G.; NOON, M.; HUDSON, M.; KAMENOU-AIGBEKAEN N.; SOSENKO, F.. Intersectionality, identity work and migrant progression from low-paid work: A critical realist approach. Gender, Work and Organization, v.27, n.6, p.1020-1039, 2020. DOI: https://doi.org/10.1111/gwao.12437

PRIMECZ, H.; KARJALAINEN, H.. Gender relations in the workplace: The experience of female managers in African harbours. International Journal of Cross Cultural Management, v.19, n.3, p.291-314, 2019.
SAIFUDDIN, S.; DYKE, L.; HOSSAIN, M. S.. Walls all around: barriers women professionals face in high-tech careers in Bangladesh. Equality, Diversity and Inclusion, v.38, n.7, p.705-726, 2019.

SALEM, R.; YOUNT, K. M.. Structural accommodations of patriarchy: Women and workplace gender segregation in Qatar. Gender, Work and Organization, v.26, n.4, p.501-519, 2019.

YAMAK, S.; ERGUR, A.; ÖZBILGIN, M. F.. Gender as symbolic capital and violence: the case of corporate elites in turkey. Gender, Work and Organization, v.23, n.2, 2016. DOI: http://doi.org/10.1111/gwao.12115

A CBPC - Companhia Brasileira de Produção Científica (CNPJ: 11.221.422/0001-03) detém os direitos materiais desta publicação. Os direitos referem-se à publicação do trabalho em qualquer parte do mundo, incluindo os direitos às renovações, expansões e disseminações da contribuição, bem como outros direitos subsidiários. Todos os trabalhos publicados eletronicamente poderão posteriormente ser publicados em coletâneas impressas sob coordenação da Sustenere Publishing, da Companhia Brasileira de Produção Científica e seus parceiros autorizados. Os (as) autores (as) preservam os direitos autorais, mas não têm permissão para a publicação da contribuição em outro meio, impresso ou digital, em português ou em tradução. 